\title{
Tratamento da doença coronariana no Brasil: um quadro que reflete a necessidade de mudança de paradigma
}

\author{
Tales de Carvalho ${ }^{1}$
}

O principal problema de saúde pública do mundo ocidental, a doença coronariana, em nosso país apresenta três grandes agravantes: manifesta-se mais precocemente, temnos colocado como líderes de procedimentos invasivos, mas não tem nos sensibilizado para uma prática séria de prevenção e reabilitação.

No banco de dados dos estudos multicêntricos com trombolíticos (EMERAS, RAPT e ISIS 4) constata-se haver no Brasil uma prevalência de $38 \%$ de infarto agudo do miocárdio (IAM) em indivíduos abaixo de 55 anos contra $28 \%$ nos demais países. Do estudo OASIS (Organization to Assess Strategies for Ischaemia Syndromes) constata-se que dos pacientes com angina instável (AI) e infarto não Q (INQ), no Brasil, são encaminhados $25 \%$ para cirurgias de revascularização miocárdica (RM) e $22 \%$ para angioplastias (ATC). Na Austrália as cifras são de 1\% e 4\%; no Canadá, de $7 \%$ e $14 \%$; na Hungria, de $5 \%$ e $5 \%$; e nos EUA, de $20 \%$ e $28 \%$, respectivamente, para RM e ATC, em pacientes com AI ou INQ. Os percentuais citados demonstram ser o Brasil um dos países que mais investe em procedimentos caros e sofisticados. Uma situação que só encontra paralelo nos EUA. Apesar dos parcos recursos destinados à saúde em nosso país, pode-se dizer que, pelo menos no que se refere à doença coronariana, não se gasta pouco. Afinal, somos o segundo país do mundo na utilização de procedimentos invasivos de revascularização do miocárdio. Em números absolutos perdemos apenas para os Estados Unidos da América do Norte. Em números relativos, considerando a população e o número de procedimentos, ocupamos o primeiro lugar. Considerando cirurgias de revascularização miocárdica e angioplastias somamos anualmente cerca de 80.000 procedimentos $^{1-4}$.

1. Presidente Eleito da Sociedade Brasileira de Medicina do Esporte; Membro da Comissão Científica do Departamento de Ergometria e Reabilitação da Sociedade Brasileira de Cardiologia; Coordenador do Programa de Prevenção e Reabilitação Cardiovascular de Santa Catarina; Membro do Comitê Técnico Científico (Atividade Física e Saúde) do Ministério da Saúde.

Endereço para correspondência:

E-mail: tales@ativanet.com.br

Rev Bras Med Esporte - Vol. 6, № 6 - Nov/ Dez, 2000
Entretanto, estudos com pacientes com doença coronariana têm questionado as vantagens desses procedimentos. Um grupo de 150 pacientes, com boa função ventricular, que recusaram cirurgia de revascularização miocárdica formalmente indicada, foi acompanhado por um período de dois a oito anos, constatando-se uma boa evolução clínica, um bom índice de sobrevida e importante remissão de sintomas. A mortalidade anual foi de $0 \%$ para doença com um ou dois vasos comprometidos e 1,3\% para os pacientes com três vasos afetados, inclusive com lesões de tronco ou equivalente a lesão de tronco de coronária esquerda. Dados muito mais favoráveis do que os decorrentes de complicações imediatas da própria cirurgia de revascularização. A sobrevida foi de $100 \%$ para os pacientes com doença de um e dois vasos e de $90 \%$ para os com doença de três vasos ou tronco de coronária esquerda. No início dos estudos, a maioria dos pacientes era sintomática (89\%), sendo $53 \%$ com angina estável e 36\% com angina instável. Controle dos sintomas ocorreu prontamente, com o início do tratamento clínico, persistindo no decorrer do estudo. $\mathrm{Na}$ última observação, $69 \%$ dos pacientes se encontravam livres de angina, $30 \%$ apresentaram angina estável e apenas $1 \%$ angina instável, refletindo uma significativa melhora. Os novos infartos foram em número de 15 (10\%), dos quais 12 não fatais. Nenhum paciente com doença de um vaso apresentou novo infarto do miocárdio. Os pacientes foram selecionados de 1977 a 1983 e, à época, os recursos terapêuticos para o tratamento clínico eram mais limitados e menores as exigências quanto aos desfechos do tratamento. Neste estudo foi considerada hipercolesterolemia somente valor igual ou superior a $250 \mathrm{mg} / \mathrm{dl}$; fumantes, somente os usuários de mais de 20 cigarros por dia, nos cinco anos precedentes; os doentes não participaram de programas de reabilitação cardíaca e pouca importância foi dada à atividade física. Outros 154 pacientes com lesão crítica da artéria descendente anterior (acima de $80 \%$ de obstrução) foram randomizados para três tipos de tratamento: angioplastia, revascularização miocárdica com artéria mamária e tratamento exclusivamente medicamentoso. Os resultados dos três grupos, no que diz respeito a desfechos maiores (infarto do miocárdio e morte), após dois anos de 
acompanhamento, foram similares. Em outro estudo relevante, que foi o acompanhamento por 22 anos de 686 pacientes randomizados para dois grupos: tratamento apenas clínico ou tratamento no qual, além dos recursos clínicos, se utilizou, também, a cirurgia cardíaca, constatou-se que sobreviveram e permaneceram livres de infarto mais pacientes no grupo que se submeteu apenas ao tratamento clínico, com $20 \%$ a menos de morte e $33 \%$ a menos de infarto ${ }^{5-7}$.

O banco de dados do OASIS (pacientes tratados com trombolíticos) permitiu o estudo de 7.987 pacientes de seis países, com angina instável ou suspeita de infarto do miocárdio sem elevação de ST, revelando incidência significativamente maior de sangramento e acidente vascular cerebral, sem vantagens em desfechos como mortalidade e incidência de infarto do miocárdio, nos pacientes tratados com métodos invasivos. São dados consistentes com os de dois estudos randomizados: o TIMI II, realizado em pacientes com infarto agudo do miocárdio, e o TIMI III, realizado em pacientes com angina instável, os quais não mostraram vantagens dos procedimentos invasivos, quando comparados com uma estratégia mais conservadora. São dados que corroboram os estudos de Parizi e Stephen. Neste último, desenvolvido em pacientes aleatoriamente encaminhados para tratamento meramente clínico ou com angioplastia, com lesões críticas residuais, após o uso de trombolíticos em evento agudo, constatou-se ausência de IAM no seguimento de um ano no grupo tratado clinicamente e incidência de $11,3 \%$ de IAM no grupo tratado com angioplastia ${ }^{8-11}$.

O tratamento da doença coronária deve ter como base a adoção de um estilo de vida que considere incremento da atividade física, reformulação de hábitos alimentares e controle do estresse, o que pode promover até mesmo regressão de aterosclerose, com importante melhora do quadro clínico e significativa interferência nos desfechos da doença. Estes recursos são suficientes para o tratamento de muitos pacientes. Para uma parcela dos pacientes, há ne-

\section{REFERÊNCIAS}

1. EMERAS collaborative group. Estudio Multicéntrico Estreptoquinasa Repúblicas de América del Sur. Lancet 1993;342:767-72.

2. RAPT investigators. Ridogrel versus aspirin patency trial. Circulation 1994;89:588-95.

3. ISIS 4 Collaborative group. Fourth international study of infarct survival. Lancet 1995;345:669-95.

4. Carvalho T. Atividade física e saúde: orientações básicas sobre atividade física e saúde para profissionais das áreas de educação e saúde. Brasília: Ministério da Saúde, Educação e Desportos, 1995.

5. Hueb W, Bellotti G, Ramires JAF, Da Luz PL, Pillegi F. Two-to eightyear survival rates in patients who refused coronary artery bypass grafting. Am J Cardiol 1989;63:155-9.

6. Hueb WA, Arie S, Oliveira SA, Bellotti G, Jatene A, Pileggi F. Randomized trial of surgery, angioplasty or medical therapy for single vessel cessidade de se acrescentar o uso de medicamentos que aliviem sintomas e reduzam um risco de complicações agudas. Qualquer paciente com o quadro clínico estabilizado, após adoção das medidas referidas, não necessitaria, a prio$r i$, de outras providências. Somente quando o quadro clínico se apresentar ainda instável, prenunciando um evento agudo, o que ocorre raramente quando os pacientes se utilizam de um tratamento clínico correto, é que se justifica a adoção de providências complementares, mais agressivas, como é o caso das cirurgias cardíacas e angioplastias. São procedimentos que devem sempre ser considerados de exceção. No Brasil, com freqüência se desrespeita esta seqüência lógica de procedimentos. É o que dizem os dados disponíveis sobre incidência de doença coronária, elevado número de procedimentos invasivos de revascularização miocárdica e a quantidade de pacientes atendidos em programas de reabilitação cardíaca. Estes programas deveriam se constituir na base do sistema. Alguns milhões de pacientes, com doença já diagnosticada, antes ou depois dos infartos do miocárdio, antes ou após as cirurgias e angioplastias, deveriam estar sendo atendidos em programas de reabilitação cardíaca, os quais não atendem em todo o país a mais do que 2.000 pacientes.

Diante da eficiência, baixo risco, baixo custo, viabilidade e aplicabilidade do tratamento clínico da doença coronária, realizado em programas de reabilitação cardíaca, é absurda a forma de investimento que privilegia quase que exclusivamente os procedimentos de alto custo, sofisticados, invasivos e muitas vezes questionáveis. A mudança de paradigmas que se impõe exige uma reformulação de conceitos científicos ultrapassados (modificação da cultura médica hegemônica), redefinição nas prioridades de investimento em saúde (revisão profunda das políticas de saúde pública) e democratização do acesso à informação. $\mathrm{Na}$ nossa experiência em Santa Catarina, este último aspecto, a mudança da cultura popular, tem sido o mais decisivo para uma mudança mais rápida ${ }^{4,12-19}$.

proximal left anterior descending artery stenosis. Results of long-term follow-up. J Am Coll Cardiol 1994;268A(760-5)

7. Peduzzi P. Twenty-two-year follow-up of VA cooperative study of coronary artery by pass surgery for stable angina. Am J Cardiol 1998;81: 1393-9.

8. Anderson HV. One-year results of the thrombolysis in myocardial infarction (TIMI) III B clinical trial. J Am Coll Cardiol 1995;26:1643-50.

9. Parizi AF. A comparison of angioplasty with medical therapy in the treatment of single-vessel coronary artery disease. N Engl J Med 1992;326: 10-6.

10. Stephen GE. Randomized trial of late elective angioplasty versus conservative management for patients with residual stenoses after thrombolytic treatment of myocardial infarction. Circulation 1992;86:1400-6.

Rev Bras Med Esporte - Vol. 6, № 6 - Nov/ Dez, 2000 
11. Aguirre FV. Early and 1-year clinical outcomes of patients evolving nonQ-wave versus Q-wave myocardial infarction after thrombolysis. Results from the TIMI II study. Circulation 1995;91:2541-8.

12. Carvalho T. Sedentarismo e doenças cardiovasculares. In: Porto CC. Doenças do coração. Prevenção e tratamento. Rio de Janeiro: Guanabara Koogan, 1998:139-41.

13. Carvalho T. Exercício físico e coração. In: Porto CC. Doenças do coração. Prevenção e tratamento. Rio de Janeiro: Guanabara Koogan, 1998: 1030-7.

14. Carvalho T. Sedentarismo o inimigo público nº 1. Rev Bras Med Esporte 1999;5:xi-xii.

15. Hambrecht R. Various intensities of leisure time physical activity in patients with coronary artery disease: effects on cardiorespiratory fitness and progression of coronary atherosclerotic lesions. J Am Coll Cardiol 1993;22:468-77.

16. Nieubauer J, Velich T, Hambrecht R, et al. Six years of intensive physical exercise and low fat diet: effects on progression of coronary artery disease. Circulation 1995;92(Suppl 1):398.

17. Ornish D. Can lifestyle changes reverse coronary artery disease? Lancet 1990;336:129-31

18. Schell DW, Myers N. Regression of atherosclerosis: a review. Progr Cardiovasc Dis 1997;34:483-96.

19. Vongvanich P. Safety of medically supervised exercise in a cardiac rehabilitation center. Am J Cardiol 1996;77:1383-5. 